\title{
Variability in data: The Society of Thoracic Surgeons National Adult Cardiac Surgery Database
}

\author{
Morgan L. Brown, MD, ${ }^{\mathrm{a}}$ Judy R. Lenoch, ${ }^{\mathrm{b}}$ and Hartzell V. Schaff, $\mathrm{MD}^{\mathrm{b}}$
}

Objective: Since 1989, data have been reported to the Society of Thoracic Surgeons National Adult Cardiac Surgery Database for quality improvement. This information is also data mined for national quality indicators, policy initiatives, and research. Such use has important limitations, because data elements cannot be verified for accuracy. We determined variability of disease etiology and operative data database elements when abstracted by untrained physician abstractors.

Methods: We selected 30 patients who underwent cardiovascular surgery from January to December 2005 (10 each of coronary artery bypass grafting, mitral valve repairs, and aortic valve and associated aortic procedures). Four abstractors ( 2 cardiothoracic residents and 2 fellows) abstracted 28 variables. Results were compared with abstraction performed by a professional abstractor.

Results: Median percentage agreement among all cases was $89 \%$ (range, $42 \%-100 \%$ ). Agreements were $94 \%$ $(28 \%-100 \%)$ for mitral valve, $84 \%(48 \%-100 \%)$ for aortic valve, and $93 \%(35 \%-100 \%)$ for coronary artery bypass grafting. Among the aortic valve group, etiology of aortic valve disease had poor agreement $(68 \%)$ because of cases in which multiple definitions could apply. Degree of valvular regurgitation also had poor agreement (median, 67\%; range, 28\%-95\%). Number of internal thoracic artery grafts and absence of significant valvular disease were reported consistently. Agreements between types of aortic valve procedure and between methods of mitral valve repair ( $65 \%$ and $83 \%$, respectively) were less than expected.

Conclusions: We found variable agreement among untrained data abstractors. This has important implications regarding interpretation of database studies with de-identified data. Without good quality control and consistent standardized definitions, aggregate data in clinical databases may be suspect. (J Thorac Cardiovasc Surg 2010;140:267-73)

The Society of Thoracic Surgeons (STS) National Adult Cardiac Surgery Database (NCD) is a North American cardiac surgical database designed to support quality improvement (http://www.sts.org). Since its development in 1989, there has been an increasing focus on obtaining and maintaining a high quality of data through on-site data managers, point-of-entry data controls, and annual national database meetings. ${ }^{1-3}$ The measures of data quality used by the STS NCD, however, are generally limited to controlling for missing data, inconsistent data, and outliers or extreme

\footnotetext{
From the Department of Anesthesiology and Pain Medicine, ${ }^{a}$ University of Alberta, Edmonton, Alberta, Canada; and Division of Cardiovascular Surgery, ${ }^{\mathrm{b}}$ Mayo Clinic, Rochester, Minn.

Supported by grant No. 1 TL1 RR024152-01 from the National Center for Research Resources (NCRR) of the National Institutes of Health (NIH) and the NIH Roadmap for Medical Research. Its contents are solely the responsibility of the authors and do not necessarily represent the official view of NCRR or NIH. Information on NCRR is available at http://www.ncrr.nih.gov/. Information on Reengineering the Clinical Research Enterprise can be obtained from http://nihroadmap.nih.gov/clinical research/overviewtranslational.asp.

Disclosures: None.

Received for publication July 15, 2009; revisions received Feb 3, 2010; accepted for publication Feb 26, 2010; available ahead of print May 24, 2010.

Address for reprints: Hartzell V. Schaff, MD, Mayo Clinic, 200 1st St SW, Rochester MN 55905 (E-mail: schaff@mayo.edu).

$0022-5223 / \$ 36.00$

Copyright (C) 2010 by The American Association for Thoracic Surgery

doi:10.1016/j.jtcvs.2010.02.052
}

data values. Only recently has there been implementation of site checking with repeated abstraction of data by data quality personnel, ${ }^{4}$ and results of these repeated abstractions have not yet been reported by the STS NCD. Our objective was to examine the amount of variability present in variables of diagnosis and operation type during data collection by untrained abstractors for the STS NCD at a single institution.

\section{MATERIALS AND METHODS}

After institutional review board approval and waiver of the need for individual patient consent, we identified patients who had undergone isolated coronary artery bypass grafting (CABG), mitral valve repair, and aortic valve or aortic procedures between January 1 and December 31, 2005 From these, a convenience sample of 10 cases was selected from each operative type, representing a variety of diagnoses and operative procedures. The year 2005 was selected because all medical records were available electronically, and the residents and fellows were not present at our clinic for any of these cases, removing the impact of recall bias. Four abstractors were identified, including 2 cardiothoracic surgical residents and 2 cardiovascular surgical fellows who were all familiar with the electronic medical record and had previous research abstraction experience. These resident and fellow abstractors were instructed in collecting variables for the STS database, including all relevant definitions provided by the STS, and were blinded to the results of the professional abstractors and those of one another. Each of the resident and fellow abstractors each reviewed al 30 patient records. These results were compared with the abstraction performed by a professional abstractor.

Data elements were identified from STS version 2.52 and included definitions of diagnoses and operation types. The variables chosen are listed in 


\section{Abbreviations and Acronyms \\ $\mathrm{CABG}=$ coronary artery bypass grafting \\ NCD $=$ National Adult Cardiac Surgery \\ Database \\ STS $=$ Society of Thoracic Surgeons}

Table 1. The variable describing valvular etiology, selected from STS version 2.35, has been removed in later versions of the core fields of the STS database but is still captured at Mayo Clinic. Resident and fellow abstractors, as well as the professional abstractor, used all information available in the electronic medical record, including operative reports, echocardiograms, cardiac catheterization reports, pathologic examinations, and any clinical notes. For internal quality control, residents and fellows also abstracted the surgeon and assistant on record for the procedure. This had $100 \%$ agreement in all cases, and these variables were not considered in further analyses.

Operations $(\mathrm{n}=30)$ were analyzed as a group as well as by separate procedure (mitral valve repair, CABG, and aortic valve or aortic procedures). Overall percentage agreements between physician abstractors and professional abstractor were calculated with the data abstractor considered as the criterion standard. In addition, Fleiss $\kappa$ values were calculated for all variables to account both for multiple abstractors and for multiple categories of possible rater responses for most variables. ${ }^{5}$ Fleiss $\kappa$ represents the degree of agreement in classification beyond that which would be expected by chance and is scored between 0 and 1. Stats Direct statistical software (version 2.6.2; StatsDirect Ltd, Altrincham, UK) was used for all calculations.

\section{RESULTS}

The median percentage agreement among all cases was $89 \%$ (range, $42 \%-100 \%$; Figure 1 ), and the $\kappa$ values are listed in Table 2. The best agreement was for variables that were not present in any patients, such as pulmonary valve stenosis and annular enlargement procedure. These both had $100 \%$ agreement, and $\kappa$ values cannot be calculated for conditions that are not present. The poorest agreement occurred in the assessment of tricuspid valve regurgitation (VDInsufT), but this was most often only 1 category of difference: none versus trivial, trivial versus mild, mild versus moderate, or moderate versus severe $(\kappa=0.206)$. This was due both to multiple sources of data for tricuspid regurgitation (transthoracic echocardiograms, transesophageal echocardiograms, and occasional cardiac catheterization or magnetic resonance imaging) and to how reports of regurgitation that included 2 categories were handled (eg, moderate to severe regurgitation). The mitral valve repair group had a median percentage agreement of $94 \%(28 \%-100 \%)$ for all fields (Figure 2$)$. The degree of mitral valve regurgitation (VDInsufM), status of the operation (Status), and number of diseased vessels (NumDisV) were collected consistently (median percentage agreements $>95 \%$ ). When looking at the type of mitral valve repair, however, there was only $83 \%$ agreement. Differences in interpretation of mitral valve repair type were most frequently due to confusion about the definition of mitral valve recon-
TABLE 1. Data elements from the Society of Thoracic Surgeons National Adult Cardiac Database assessed for variability

\begin{tabular}{ll}
\hline Variable name & \multicolumn{1}{c}{ Variable meaning } \\
\hline Surgeon & Primary surgeon \\
Assistant & Primary assistant \\
VDStenA & Aortic valve stenosis \\
VDStenM & Mitral valve stenosis \\
VDStenT & Tricuspid valve stenosis \\
VDStenP & Pulmonary valve stenosis \\
VDInsufA & Aortic valve insufficiency \\
VDInsufM & Mitral valve insufficiency \\
VDInsufT & Tricuspid valve insufficiency \\
VDInsufP & Pulmonary valve insufficiency \\
VDEtiolA & Aortic valve etiology \\
VDEtiolM & Mitral valve etiology \\
VDEtiolT & Tricuspid valve etiology \\
VDEtiolP & Pulmonary valve etiology \\
ReopRsn & Reason for reoperation \\
Status & Urgency of operation \\
AnlrEnl & Annular enlargement procedure \\
OpAortic & Aortic valve procedure type \\
OpMitral & Mitral valve procedure type \\
ONCAoAn & Aortic aneurysm \\
ONCAsc & Ascending aortic aneurysm \\
ONCArch & Aortic arch aneurysm \\
NumDisV & No. of diseased vessels \\
LMainDis & Left main coronary artery disease \\
IMAArtUs & Internal thoracic arteries used \\
RadArtUs & Radial arteries used \\
DistArt & No. of distal anastomoses with arterial conduits \\
DistVein & No. of distal anastomoses with venous conduits \\
\hline &
\end{tabular}

struction with or without annuloplasty. Although the STS definition mentions that annuloplasty requires placement of an annuloplasty ring, there were cases in which a suture annuloplasty was performed, such as a Kay stitch, and these were coded as annuloplasty by some of the untrained abstractors.

In the aortic valve or aortic procedure group, the percentage agreement ranged between $48 \%$ and $100 \%$, with a median value of $84 \%$ for all fields (Figure 3). Variables of aortic stenosis (VDStenA) and replacement of the aortic arch (ONCArch) had median percentage agreements of $93 \%$ and $93 \%$, respectively. Among patients in the aortic valve or aortic procedure group, the lowest agreement was seen in the degree of mitral valve regurgitation (VDInsufM), which was most commonly misclassified by only 1 category. If VDInsufM was dichotomized to none, trivial, or mild versus moderate or severe, the $\kappa$ value was 0.970 , but if VDInsufM was dichotomized to none or trivial versus mild, moderate, or severe, the $\kappa$ value decreased to 0.756 . It was notable that the type of aortic valve procedure (OpAortic) had a median agreement of only $65 \%$. This was primarily due to variability regarding the coding of root reconstruction with valve sparing versus resuspension aortic valve with 


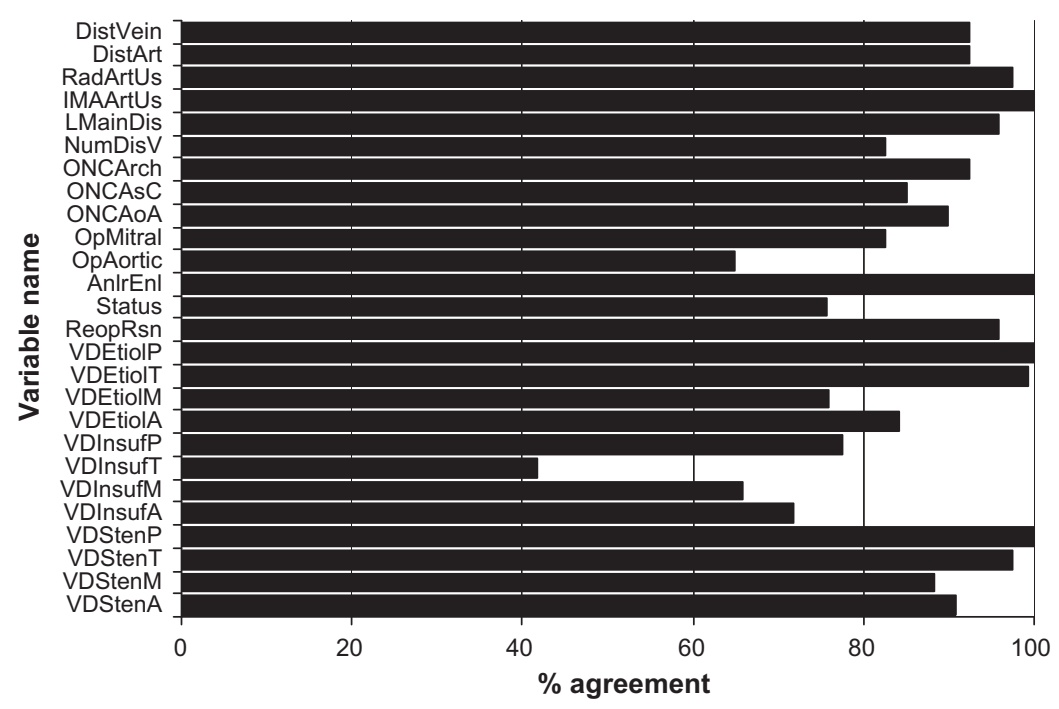

FIGURE 1. Percentage agreement for all definitions (given in Table 1) in all operations.

replacement of ascending aorta versus resuspension aortic valve without replacement of ascending aorta.

Data for patients undergoing $\mathrm{CABG}$ had a median agreement of $93 \%$ (range, $35 \%-100 \%$ ) for all fields (Figure 4).

TABLE 2. Fleiss $\kappa$ values for all patients with records abstracted

\begin{tabular}{lc}
\hline \multicolumn{1}{c}{ Variable } & Fleiss $\boldsymbol{\kappa}$ \\
\hline Aortic valve stenosis & $0.62 \pm 0.06$ \\
Mitral valve stenosis & $0.13 \pm 0.06$ \\
Tricuspid valve stenosis & $-0.02 \pm 0.06$ \\
Pulmonary valve stenosis & NA \\
Aortic valve insufficiency & $0.50 \pm 0.03$ \\
Mitral valve insufficiency & $0.56 \pm 0.04$ \\
Tricuspid valve insufficiency & $0.21 \pm 0.04$ \\
Pulmonary valve insufficiency & $0.04 \pm 0.04$ \\
Aortic valve etiology & $0.66 \pm 0.04$ \\
Mitral valve etiology & $0.66 \pm 0.03$ \\
Tricuspid valve etiology & $-0.02 \pm 0.06$
\end{tabular}

Pulmonary valve etiology

Reason for reoperation

Urgency of operation

Annular enlargement procedure

Aortic valve procedure type

Mitral valve procedure type

Aortic aneurysm

Ascending aortic aneurysm

Aortic arch aneurysm

No. of diseased vessels

Left main coronary artery disease

Internal thoracic arteries used

Radial arteries used

No. of distal anastomoses with arterial conduits

No. of distal anastomoses with venous conduits

Fleiss $k$ values (listed as $k+\mathrm{SE}$ ) are influenced by number of categories or ratings possible for any variable. In addition, $\kappa$ values are less reliable in cases of rare events. Fleiss $K$ values cannot be calculated if there are no events in the variable of interest. $N A$, Not applicable.
Importantly, internal thoracic artery usage (IMAArtUs) had a 100\% agreement, and numbers of distal vessels (DistArt and DistVein) had agreements greater than $90 \%$. Left main disease had an agreement of $88 \%$ and number of diseased vessels had an agreement of $78 \%$, however, both of which are less than would be expected. The urgency of operation had only a $68 \%$ agreement, despite lengthy definitions provided by STS.

\section{DISCUSSION}

In our study, variables abstracted for the STS NCD had a median percentage agreement of $89 \%$, but there was a wide range between $42 \%$ and $100 \%$. These results demonstrate the potential variability when abstracting data for institutional registries such as the STS NCD. There are 3 potential problems with the abstraction of clinical data: the quality of the available source material, the definitions and timing of variables captured, and the interpretation of the source materials and definitions by an abstractor.

The quality of the available source material is an important issue. At many institutions, there may be inconsistent availability and completeness of echocardiographic and angiographic reports, operative reports, pathology reports, and clinical records. At Mayo Clinic, there is a single universal chart for the patient. In other practices, however, a given patient may have data scattered among many charts located in different physician offices, divisions, or departments, and the most important current data may not be available to the abstractor.

In addition, clinical data may change during the duration of a hospital admission. In the materials provided by STS, there are no guidelines in the definitions or in the frequently asked questions section regarding how source materials should be prioritized and the timing of when data should be extracted 


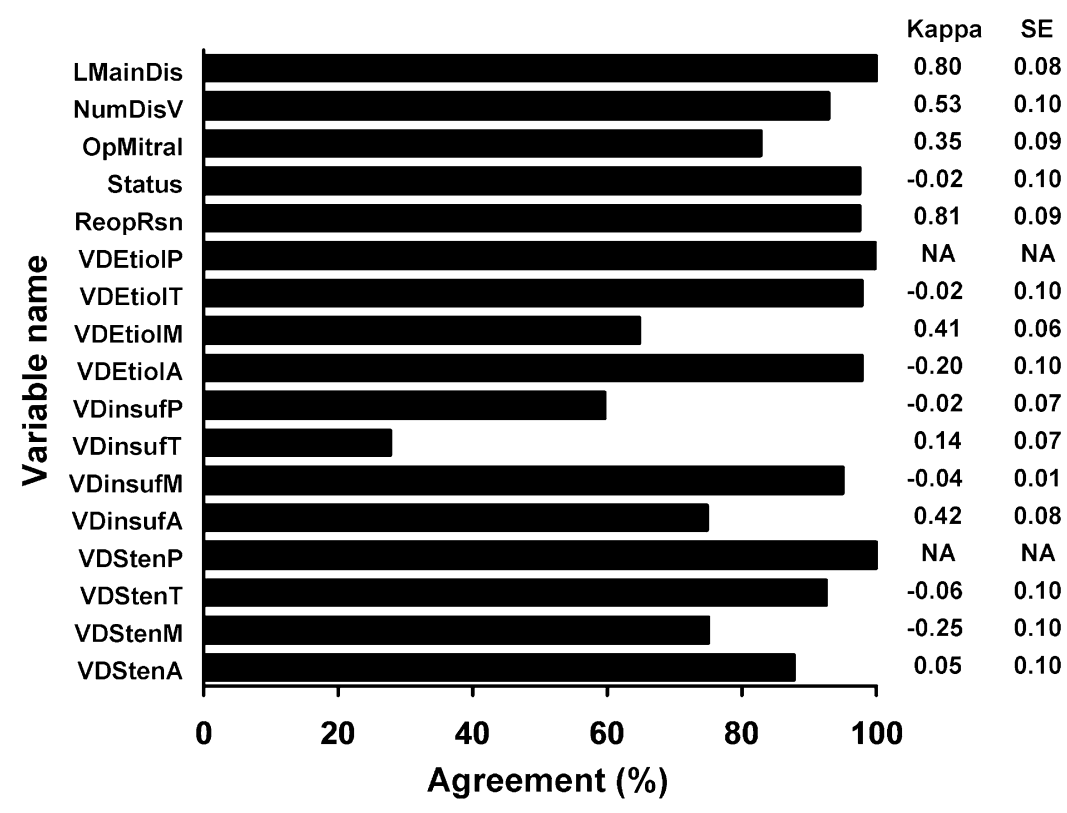

FIGURE 2. Percentage agreement for all definitions (given in Table 1) collected for patients who underwent mitral valve repair procedures. $\kappa$ values and SEs are listed for each variable. NA, Not applicable.

in the patient's medical history. ${ }^{1-3}$ For example, a patient might be admitted with severe congestive heart failure and severe mitral and tricuspid valve regurgitation, but adequate diuresis might subsequently reduce tricuspid regurgitation to the category of mild. If such a patient undergoes mitral valve and tricuspid valve repair, there are no guidelines with regard to the appropriate time to abstract data on the degree of tricuspid valve regurgitation. Individual centers may have internal consistency in these matters, but without clarification from the STS, data may not be comparable among centers in research studies.

The STS has a database committee that works to develop precise and clear definitions for the STS NCD. In addition, the STS provides a frequently asked questions file, and there is a contact at the central office to ask questions. As our study has demonstrated, however, there remains some confusion regarding definitions. Some confusion, for example about the fields regarding valvular etiology or reason for

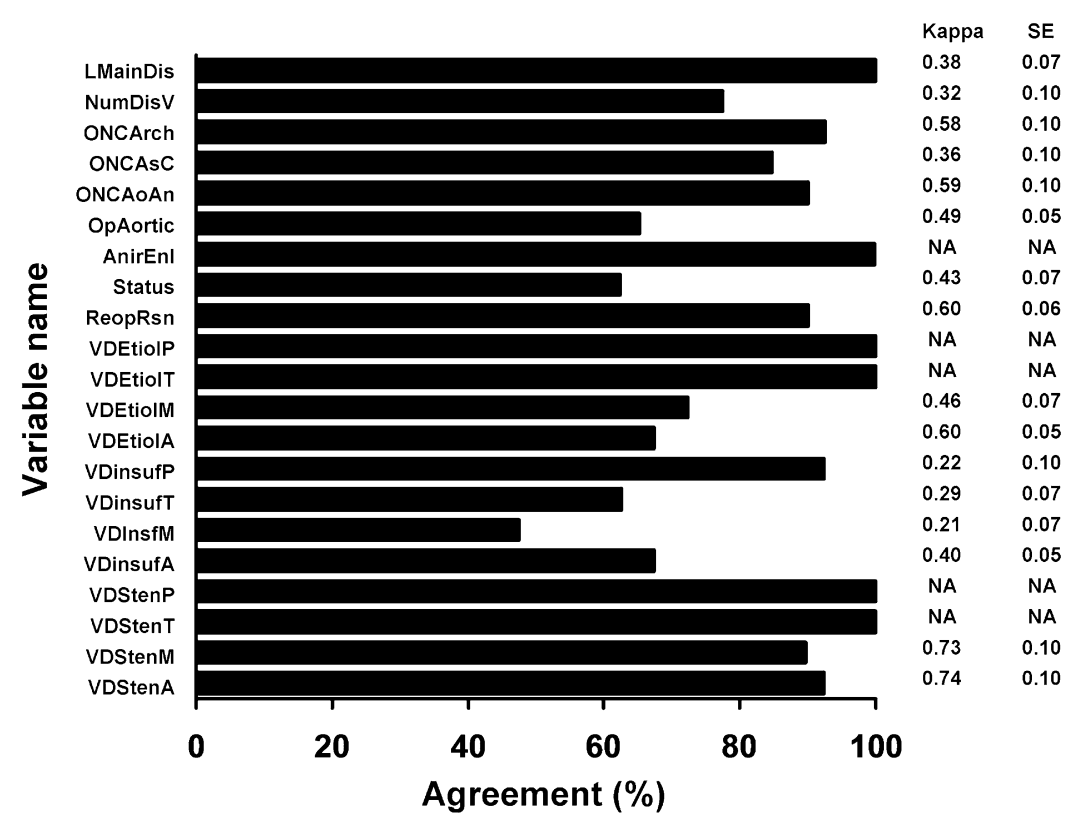

FIGURE 3. Percentage agreement for all definitions (given in Table 1) collected for patients who underwent aortic valve or aortic procedures. $\kappa$ values and SEs are listed for each variable. $N A$, Not applicable. 


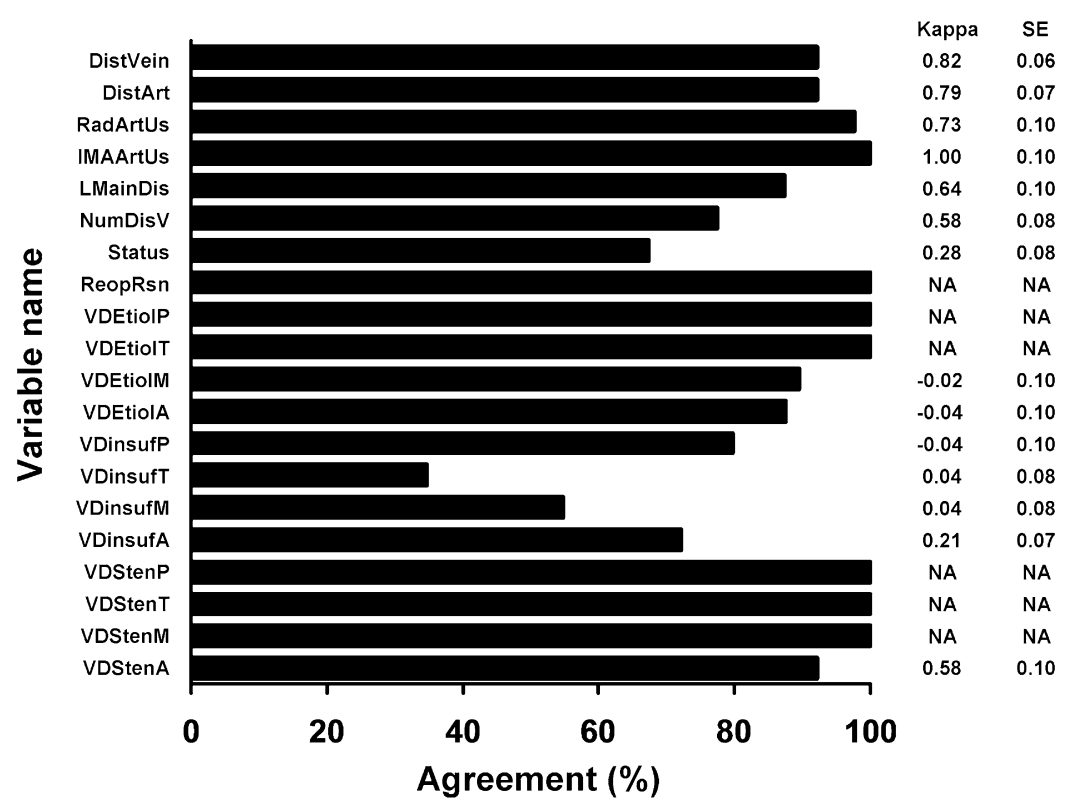

FIGURE 4. Percentage agreement for all definitions (given in Table 1) collected for patients who underwent coronary artery bypass grafting procedures. $\kappa$ values and SEs are listed for each variable. $N A$, Not applicable.

reoperation, is due to the exclusionary nature of the question, in which only a single response may be given even though many (such as rheumatic, calcific, and congenital for a stenotic bicuspid aortic valve) may be appropriate. Other disagreements regarding definitions are due to the complex nature of cardiovascular surgery, such as aortic valve and root procedures. There would be debate among surgeons themselves as to whether the ascending aorta is replaced if a surgeon performs an aortic root replacement. And how are patients categorized who undergo replacement of the ascending aorta and noncoronary sinus? Definitions may be able to be further clarified in the STS NCD to ensure consistent data collection, and the variability present in data elements should be assessed and made available.

The interpretation of source materials and definitions by abstractors is a critical step in the acquisition of accurate data. At Mayo Clinic, we have a trained data manager and 4 data abstractors who work full-time on collection of data and have accumulated decades of experience. They have created additional "data rules" for themselves to deal with many of these issues of data interpretation. They interact with each other when needed and can also consult the supervisor. The chair of the division of cardiovascular surgery also meets with the supervisor regularly to resolve issues of data interpretation. Our study was not designed to examine internal consistency of our data abstractors; we believe that we already achieve this through frequent education and the development of in-house "data rules." Our study does demonstrate, however, that with available definitions the use of untrained abstractors, even those with experience in cardiovascular surgery, may lead to considerable misclas- sification. This underlines not only the importance of providing not only clear and consistent definitions but also the need for formal abstractor training and certification, and perhaps recertification.

Recently Clarke and associates ${ }^{4}$ evaluated quality of data contained in the STS congenital cardiac surgery database. Five randomly selected centers with a minimum of 30 congenital operations in 2006 were selected for repeated abstraction by independent abstractors, including a database manager and cardiac surgeon. Administrative data had a percentage agreement ranging between $87 \%$ and $97 \%$, and agreement for intraoperative data ranged between $94 \%$ and $100 \%$. Categorization of the primary procedure had an agreement of only $72 \%$, however, and that of the primary diagnosis had an agreement of $74 \%$. It is probable that diagnosis and procedure identification are much more difficult in congenital cardiac surgery than in adult acquired cardiac surgery. Our study, however, did show an overall agreement of $89 \%$ for adult cardiac surgery diagnoses and operative procedure types, which is comparable to the results from the congenital STS database.

One may question the necessity of obtaining such a high degree of accuracy in a voluntary registry database. A recent study deliberately miscoded a database with missing data, changes in vital status, and misclassification of operative procedures; the investigators found that even small amounts of data error $(1 \%-5 \%)$ could lead to misleading analyses. ${ }^{6}$ In that study of congenital cardiac surgical patients, misclassification of low-risk procedures as high-risk procedures led to a lower calculated mortality, whereas high-risk operations misclassified as low-risk operations led to a higher calculated 
mortality. If these results were to be translated into large databases, there would potentially be a great amount of error, which could be reflected in both the assessment of quality of an institution and the ability to conduct research.

Another study of isolated CABG procedures examined the rates of error within a single institution's STS database, including risk factors, operative mortality, major complications, and other outcomes. Herbert and coworkers ${ }^{7}$ found complete correlation in only $2 \%$ of cases, 1 to 10 discrepancies in $82 \%$ of cases, and more than 10 fields of disagreement in $17 \%$ of cases. ${ }^{7}$ This study did not examine other types of operations or etiology of disease. The assessment of accuracy of this database was performed by a database supervisory person who may have followed internal rules or guidelines, and thus may not have demonstrated the same variability as if they had used "untrained" abstractors.

The STS's database has undertaken several initiatives to ensure the quality of data. Most of these efforts, however, have been limited to logic checking, missing data, and outliers. ${ }^{8}$ An example of logic checking would include patient death during the initial hospitalization with a blank date of death; software prevents record completion until the date is entered. In addition, data managers at each submitting site are given data quality reports noting inconsistent data, missing data, and extreme data. Centers may resubmit their data as many times as required. None of these measures, however, assess the accuracy of the data submitted.

There have been comparisons of data submitted to the STS database by all centers in the state of Iowa and data available in administrative datasets such as the Medical Provider and Analysis Review Part A public use data files. ${ }^{9}$ In an analysis, Welke and coworkers ${ }^{9}$ found that there were more cases of isolated $\mathrm{CABG}$ operations entered in the STS NCD than in the Medical Provider and Analysis Review database. Welke and coworkers ${ }^{9}$ suggested that institutions are submitting more complete data to STS NCD than to other administrative databases; however, this was a crude assessment. Mack and colleagues ${ }^{10}$ similarly examined the correlation between administrative data and clinical data from the Medical City Hospital in Dallas. Their study found poor agreement between databases but attributed this to calendar year versus fiscal year, populations reported, date used for the patient record captured, and the definition of mortality. These studies are examples of the variability in the completeness of data and the comparability of data among databases, but they did not assess the variability present within individual data elements in the STS NCD. Variability among databases draws into question the reliability of the content of each database being compared.

The STS has begun to conduct national audits on the accuracy of the data in the NCD. Their stated goal is to audit 20 centers per year for 62 data elements in 20 cases of isolated CABG. Preliminary results were presented at the Advances in Quality and Outcomes Conference in October 2008 on
140 cases audited at 17 centers in 2008, along with results from an audit in 2007. Variables that had perfect agreement in both 2007 and 2008 included age, sex, admission date, discharge date, and date of operation. At least 22 variables, however, had less than $90 \%$ agreement in either 2007 or 2008. Unfortunately, the patient status at 30 days (dead or alive) was only in agreement in $83 \%$ of cases in 2008 . This is of great concern, because " 30 -day mortality" or "operative mortality" is the most common outcome used for both quality indicators and research. It is notable that there was an average increase in the percentage agreement of $33 \% \pm 22 \%$ from 2007 to 2008 . There were no data given, however, on the method of selection of cases at an institution, the selection of the institutions for the audit, the types of original cases, and the distribution of variables therein. Thus although the results provide reason for concern, it is difficult to interpret them in a broader context. It is to be hoped that this auditing process will be expanded, and the results should be made available to all participating institutions. This should be done in a nonpunitive manner, but the results may significantly affect the cardiac surgical community's perception of quality control measures and research done with the database. The STS NCD has begun to implement a strategy of a "virtual patient." ${ }^{11}$ In this situation, sample records are abstracted by data managers at various centers. These results of this "virtual patient" abstraction can be used to determine the adequacy of definitions. There are, however, no publicly available data concerning these results. It would also be important for the STS to provide more detailed guidelines outlining where and when data should be collected from the patient record. Recording the date and precise source of the data would allow more accurate comparisons.

Our study is limited by using a single institution with a limited number of abstracted cases, and our resultant confidence intervals were large. Our results may also be dependent on the distribution of operations that were selected. In addition, our abstractors were cardiac surgeons in training, who could arguably be either underqualified or overqualified to complete this type of data abstraction. The STS advocates point-of-care data entry, however, and suggests in its participation manual that residents, perfusionists, and nurse clinicians contribute to data collection. ${ }^{1}$ We also limited our abstraction to diagnostic and operative variables and did not assess preoperative risk factors, outcome measures, or the completeness of the number of cases abstracted, because others have specifically focused on the abstraction of these variables. ${ }^{4,7,10}$

The STS NCD is a powerful tool in cardiovascular surgery for quality assessment and clinical research. We demonstrated some of the potential variability in the abstraction of data for this national database. Caution must be exercised when using data from the STS NCD, or any other clinical data set, without knowledge of the variability of the data contained therein. 


\section{References}

1. Society of Thoracic Surgeons [Internet]. Chicago: The Society; c2010 [updated 2009 Jan 12; cited 2009 Feb 19]. The Society of Thoracic Surgeons STS National Database and Outcomes Program Participation Manual [14 pp]. Available from: http://www.sts.org/documents/pdf/ndb/Database_Manual_2009.pdf

2. The Society of Thoracic Surgeons [Internet]. Chicago: The Society; c2010 [cited 2009 Feb 17]. The Society of Thoracic Surgeons, Database Manager Section [about 1 index screen]. Available from: http://www.sts.org/sections/stsnationaldatabase/ datamanagers/

3. The Society of Thoracic Surgeons [Internet]. Chicago: The Society; c2010 [updated 2006 Oct 27; cited 2009 Feb 17]. STS Adult Cardiac Database Training Manual, version 2.52 [about 1 index screen]. Available from: http://www. sts.org/sections/stsnationaldatabase/datamanagers/adultcardiacdb/datacollection/ articles/article1.html

4. Clarke DR, Breen LS, Jacobs ML. Verification of data in congenital cardiac surgery. Cardiol Young. 2008;18:177-87.

5. Fleiss JL. Measuring nominal scale agreement among many raters. Psychol Bull. 1971;76:378-81.
6. Gallivan S, Stark J, Pagel C, Williams G, Williams WG. Dead reckoning: can we trust estimates of mortality rates in clinical databases? Eur J Thorac Surg. 2008; 33:334-40.

7. Herbert MA, Prince SL, Williams JL, Magee MJ, Mack MJ. Are unaudited records from an outcomes registry database accurate? Ann Thorac Surg. 2004; 77:1960-4.

8. The Society of Thoracic Surgeons [Internet]. Chicago: The Society; c2010 [updated 2008 Apr 30; cited 2009 Feb 17]. STS Adult Cardiac Surgery Database v2.61 Consistency Edits and Checks [3 pp]. Available from: http://www.sts.org/ documents/pdf/ndb/Training_Manual_Consistency_Edits_043008_\%282\%29. pdf

9. Welke KF, Ferguson TB Jr, Coombs LP, Dokholyan RS, Murray CJ, Schrader MA, et al. Validity of the Society of Thoracic Surgeons National Adult Cardiac Surgery Database. Ann Thorac Surg. 2004;77:1137-9.

10. Mack MJ, Herbert M, Prince S, Dewey TM, Magee MJ, Edgerton JR. Does reporting of coronary artery bypass grafting from administrative databases accurately reflect actual clinical outcomes? J Thorac Cardiovasc Surg. 2005;129:1309-17.

11. Vahl CF, Carl I, de Simone R, Meinzer HP, Thomas G, Hagl S, et al. [Calibration of clinical databanks with "virtual patients"']. Z Cardiol. 1997;86:35-41. German.

\title{
COMMENTARY
}

\section{Data variability and validity: The elephant in the room}

\author{
Gary L. Grunkemeier, PhD, and Anthony P. Furnary, MD
}

The Society of Thoracic Surgeons (STS) National Adult Cardiac Surgery Database (NCD), begun in 1989, has found almost universal acceptance, with more than 1000 cardiac surgery programs currently participating. Cardiac surgery has been a leader in this effort, with worldwide acknowledgment, and is no doubt the envy of other surgical specialties. Only now, 20 years later, for example, is orthopedic surgery attempting to create a national registry, motivated by a 4-year, \$12 million grant from the Agency for Healthcare Research and Quality. ${ }^{1}$ Not only is cardiac surgery ahead of the pack, it is far ahead.

\section{BROWN AND COLLEAGUES}

This issue of the Journal contains an article questioning the NCD data validity. ${ }^{2}$ The objective of this article was "to determine the variability of disease etiology and operative data elements in the STS database when abstracted by untrained physician abstractors." These untrained abstracters, 4 cardiac residents and fellows, assessed 28 objec-

\footnotetext{
From the Providence Health \& Services Cardiovascular Disease Study Group, Portland, Ore.

Received for publication April 12, 2010; accepted for publication April 20, 2010. Address for reprints: Gary Grunkemeier, PhD, 9155 SW Barnes Rd, Suite LL33,

Portland, OR 97225 (E-mail: gary.grunkemeier@providence.org).

J Thorac Cardiovasc Surg 2010;140:273-5

0022-5223/\$36.00

Copyright (c) 2010 by The American Association for Thoracic Surgery

doi:10.1016/j.jtcvs.2010.04.018
}

tively quantifiable preoperative and intraoperative variables. The result was "variable agreement among untrained data abstractors in the STS database"; specifically, there was agreement only approximately $89 \%$ (median) of the time (range $42 \%-100 \%$ ).

The STS urges participants to acquire a part-time or fulltime specialist to manage the NCD, a Data Manager (DM), and provides description of the qualifications for the job and ideas of where to look for a suitable candidate. ${ }^{3}$ So, because the STS database is populated primarily with the results from DMs, of more interest would be a cross-validation study among DMs, not untrained physicians. Apparently there are 5 DMs at Mayo (Brown and colleagues ${ }^{2}$ use the term "Professional Data Abstractor"' in this article, but we use DM in conformance with STS terminology). It would have been of more relevance to measure the agreement among the DMs. If those results were not consistent, we could conclude that STS needs to make its data definitions more clear or publish more in-depth data abstraction rules.

\section{PROVIDENCE HEALTH \& SERVICES EXPERIENCE}

We represent a collaborative of all 12 cardiac surgery units in the Providence Health \& Services hospital system, located in 5 western states. Since 1997, we have collected data prospectively on a common data form, sent the data to a coordinating center for merging and auditing, and 